\title{
Clinical and Neuroimaging Correlation in Patients with COVID-19
}

\author{
(D).C. Yoon, (D) Kuch, DM. Lang, DB.P. Applewhite, (DM.D. Li, (D)W.A. Mehan, Jr, (DT.M. Leslie-Mazwi, and (D) S.P. Rincon
}

\begin{abstract}
BACKGROUND AND PURPOSE: Coronavirus disease 2019 (COVID-19) is increasingly being recognized for its multiorgan involvement, including various neurological manifestations. We examined the frequency of acute intracranial abnormalities seen on CT and/or MR imaging in patients with COVID-19 and investigated possible associations between these findings and clinical parameters, including length of hospital stay, requirement for intubation, and development of acute kidney injury.
\end{abstract}

MATERIALS AND METHODS: This was a retrospective study performed at a large academic hospital in the United States. A total of 641 patients presented to our institution between March 3, 2020, and May 6, 2020, for treatment of coronavirus disease 2019, of whom, 150 underwent CT and/or MR imaging of the brain. CT and/or MR imaging examinations were evaluated for the presence of hemorrhage, infarction, and leukoencephalopathy. The frequency of these findings was correlated with clinical variables, including body mass index, length of hospital stay, requirement for intubation, and development of acute kidney injury as documented in the electronic medical record.

RESULTS: Of the 150 patients, 26 (17\%) had abnormal CT and/or MR imaging findings, with hemorrhage in 11 of the patients (42\%), infarction in 13 of the patients (50\%), and leukoencephalopathy in 7 of the patients $(27 \%)$. Significant associations were seen between abnormal CT/MR imaging findings and intensive care unit admission $(P=.039)$, intubation $(P=.004)$, and acute kidney injury $(P=.030)$.

CONCLUSIONS: A spectrum of acute neuroimaging abnormalities was seen in our cohort of patients with coronavirus disease 2019, including hemorrhage, infarction, and leukoencephalopathy. Significant associations between abnormal neuroimaging studies and markers of disease severity (intensive care unit admission, intubation, and acute kidney injury) suggest that patients with severe forms of coronavirus disease 2019 may have higher rates of neuroimaging abnormalities.

ABBREVIATIONS: COVID-19 = coronavirus disease 2019; SARS-CoV-2 = Severe Acute Respiratory Syndrome coronavirus 2

S evere Acute Respiratory Syndrome coronavirus 2 (SARS-CoV2) infection, which causes coronavirus disease 2019 (COVID19), has been shown to have multiorgan manifestations, with involvement of the lungs, heart, kidneys, liver, and CNS. ${ }^{1-10}$ Emerging small cohort studies have demonstrated myriad neuroimaging manifestations related to COVID-19, including stroke, hemorrhagic necrotizing encephalitis, and leukoencephalopathy. ${ }^{6,9-12} \mathrm{~A}$ previous study by Mao et $\mathrm{al}^{5}$ demonstrated a high rate of neurologic

Received May 29, 2020; accepted after revision June 16.

From the Departments of Radiology (B.C.Y., K.B., M.L., B.P.A., M.D.L., W.A.M., Jr., S.P.R.) and Neurosurgery and Neurology (T.M.L.-M.), Massachusetts General Hospital, Harvard Medical School, Boston, Massachusetts.

Please address correspondence to Byung C. Yoon, MD, Department of Radiology, Massachusetts General Hospital, 55 Fruit St, Boston, MA 02114; e-mail: byoon@mgh.harvard.edu; @MghNeuroRad

- Indicates open access to non-subscribers at www.ajnr.org

http://dx.doi.org/10.3174/ajnr.A6717 symptoms in patients with COVID-19 and severe respiratory disease. A recent study by Jain et al $^{13}$ also described a spectrum of neuroimaging findings associated with COVID-19 and found that patients with acute strokes had a higher mortality risk.

However, it remains unknown exactly which clinical parameters are associated with intracranial abnormalities, in part, because patients with COVID-19 can present with a wide spectrum of laboratory and clinical findings of varying severity. Our aim was to evaluate the frequency of neuroimaging findings in patients with COVID-19, define the types of abnormality present, and investigate possible associations between neuroimaging abnormalities and different clinical parameters. Our hypothesis was that the presence of intracranial abnormalities, such as intracranial hemorrhage, infarction, and leukoencephalopathy, would be associated with clinical parameters suggestive of more severe disease, such as length of hospital stay, intensive care unit admission, and 

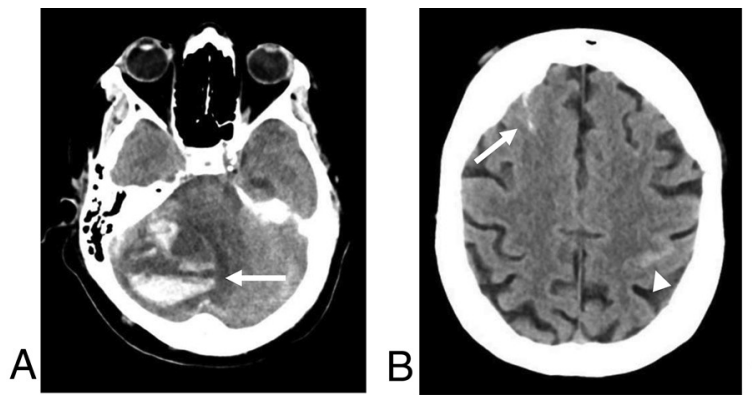

FIG 1. Examples of intracranial hemorrhages in patients with COVID-19. A, An axial noncontrast CT of a 42-year-old man demonstrates an acute intraparenchymal hematoma in the right cerebellar hemisphere, with severe mass effect and effacement of the fourth ventricle; multiple areas of layering hemorrhage are seen within the hematoma (arrow), suggestive of an underlying coagulopathy. B, Noncontrast CT of an 84-year-old woman after a fall shows scattered, curvilinear hyperdensities along the right superior frontal sulcus and left central sulcus, most consistent with acute subarachnoid hemorrhage. C, SWI of a 41-year-old man demonstrates scattered foci of susceptibility effect in the bilateral frontal and parietal lobes (arrow), most consistent with microhemorrhages.

intubation. Given that acute kidney injury in patients with COVID-19 has been reported to be associated with vascular injury (thrombi and endothelial damage) ${ }_{1}^{14}$ we also hypothesized that the presence of acute kidney injury may be associated with a higher incidence of cerebrovascular abnormalities.

\section{MATERIALS AND METHODS Study Cohort}

This was a retrospective institutional review board-approved study performed at a large academic hospital in the United States. Between March 3, 2020, and May 6, 2020, a total of 641 patients received medical care at our hospital for COVID-19. Included in our study were all patients at our hospital with documented SARS-CoV-2 infection diagnosed by reverse transcriptase-polymerase chain reaction and who underwent $\mathrm{CT}$ and/or MR imaging for the evaluation of neurologic symptoms. Exclusion criteria included patients with suspected COVID-19 who ultimately proved to be COVID-19-negative by the combination of negative reverse transcriptase-polymerase chain reaction results with negative clinical and thoracic imaging criteria, and patients with COVID-19 who underwent CT and MR imaging with substantial artifact, precluding diagnostic assessment.

\section{Clinical Data}

Basic demographic information, including age, sex, and body mass index, was obtained from the electronic medical record and recorded for each patient. Clinical data, including length of hospital stay, need for intensive care unit admission, need for intubation, clinical diagnosis of acute kidney injury, and death, were recorded for each patient. The indication for neuroimaging evaluation was recorded for each patient. The length of stay was recorded up to May 19, 2020.

\section{Imaging Technique}

All CT examinations were obtained on 64-section multidetector row CT scanners with protocols that included noncontrast CT of the head and CT angiograms of the head and neck. CT

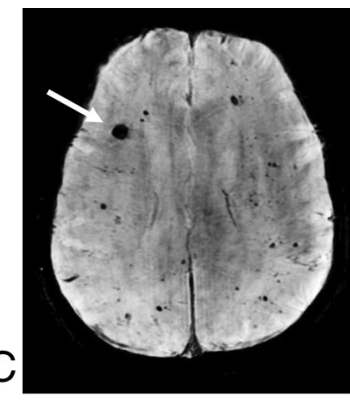

examinations were performed on either a Revolution CT (GE Healthcare) or a Force CT (Siemens) scanner. Noncontrast CT studies of the head were axially acquired (120-kV/auto$\mathrm{mAs})$ at $5-\mathrm{mm}$ - and $1.25-\mathrm{mm}$-thick slices, and imaging was obtained from the level of the skull base to the vertex. Soft tissue and bone algorithms were available for review with $1.5-2-\mathrm{mm}$ thick/interval coronal and sagittal reconstructions.

CT angiogram images of the neck were obtained from the level of the aortic arch through the superior orbital rim with 0.625-1.25-mm-thick slices (120-kVp/auto-mAs), after a dose of $80-100 \mathrm{~mL}$ of iohexol (Omnipaque; GE Healthcare) or iopamidol (Isovue; Bracco), $370 \mathrm{mg} /$ $\mathrm{mL}$ administered by a power injector at a rate of $4-5 \mathrm{~mL} / \mathrm{s}$. Coronal and sagittal reformats with 2 -mm-thick slices were reconstructed. MR imaging examinations of the brain were obtained on either a 1.5T Signa scanner (GE Healthcare) or a 3T Skyra scanner (Siemens).

MR imaging sequences performed included axial DWI, axial T2 FLAIR, axial T2WI, axial SWI (susceptibility-weighted imaging), and sagittal T1WI. Intravenous contrast was used in a subset of studies.

\section{Image Evaluation}

All CT and MR imaging examinations obtained during the hospitalization of patients for COVID-19 were reviewed by 2 neuroradiologists. Intracranial abnormalities, including the presence of hemorrhage, acute or subacute infarction, and/or leukoencephalopathy (Figs 1 and 2) were recorded. For intracranial hemorrhages, the compartment of intracranial hemorrhage (SAH, subdural, epidural, intraventricular, or parenchymal) was defined. The relative size of parenchymal hemorrhage was recorded (microhemorrhage versus lobar hemorrhage). Microhemorrhages were defined as foci of susceptibility effect that were not evident on other sequences such as T1WI or T2WI. The imaging studies were also evaluated for the presence of acute or subacute infarctions with the location and vascular territory of the infarct recorded. The presence of acute WM injury and/or leukoencephalopathy was also recorded, defined as restricted diffusion with corresponding T2 FLAIR hyperintensity within the WM. ${ }^{15,16}$

\section{Data Analysis}

A Fisher exact test was performed to evaluate for potential associations between the frequency of recorded neuroimaging abnormalities and clinical parameters, including the length of hospital stay, intubation status, and acute kidney injury. A 2 -tailed $t$ test was performed to evaluate for significant differences in patient age and body mass index for patients with negative versus positive neuroimaging studies. $P<.05$ was considered statistically 
significant. GraphPad Prism (GraphPad Software) was used for statistical analysis.

\section{RESULTS}

A total of 150 patients with COVID-19 diagnosed by reverse transcriptase-polymerase chain reaction underwent neuroimaging at our institution. Among these, 141 patients had a CT scan, 21 patients had an MRI, and 31 patients had both CT and MR examinations. None of the patients was excluded from the study for suboptimal imaging. One of the patients had an MR
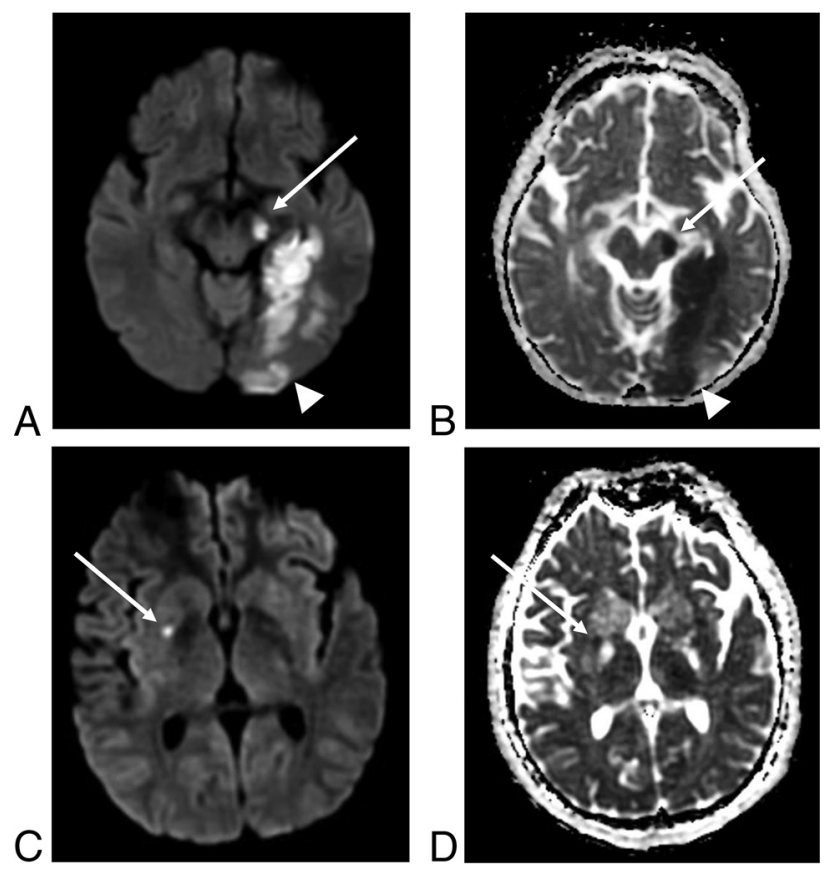

FIG 2. Examples of acute infarcts in patients with COVID-19. Axial DWI $(A)$ and corresponding apparent diffusion coefficient map $(B)$ of a 50-year-old woman demonstrate restricted diffusion related to an acute left posterior cerebral artery territory infarction. The left temporo-occipital region (arrowhead), left hippocampus, and the left cerebral peduncle (arrow) are involved. Axial DWI (C) and ADC (D) images of a 24-year-old man shows a small, subtle focus of restricted diffusion in the right putamen (arrow), most consistent with an acute infarct. examination that was motion degraded, but the imaging quality was deemed adequate to rule out acute intracranial abnormalities. The same patient had a follow-up MR imaging 16 days after the motion-degraded, initial MR study, which also did not demonstrate any acute or subacute intracranial abnormalities. Twentysix patients (17\%) had an abnormal neuroimaging study; the remaining 124 patients (83\%) had no acute intracranial abnormality. The mean age $(+/-$ standard deviation) of our cohort was $63.6 \pm 16$ years, with a range of $22-96$ years. There were 98 men and 52 women enrolled in the study (Table 1).

Table 2: Summary of clinical presentation, imaging indications, and findings

\begin{tabular}{ll}
\hline \multicolumn{1}{c}{ Clinical Presentation } & $n / N(\%)$ \\
\hline Respiratory symptoms ${ }^{\text {a }}$ & $21 / 26(81)$ \\
Fever & $7 / 26(27)$ \\
Headache & $2 / 26(8)$ \\
Fall & $2 / 26(8)$ \\
Stroke symptoms & $2 / 26(8)$ \\
Multiple concerns & $12 / 26(46)$ \\
Imaging indications & \\
$\quad$ Altered mental status & $14 / 26(54)$ \\
Concern for infarct & $8 / 26(31)$ \\
Concern for intracranial hemorrhage & $1 / 26(4)$ \\
Hypoxia & $1 / 26(4)$ \\
Seizure & $1 / 26(4)$ \\
Headache & $1 / 26(4)$ \\
Imaging findings & \\
Intracranial hemorrhage & $11 / 26(42)$ \\
$\quad$ SAH & $2 / 11(18)$ \\
$\quad$ Intraparenchymal hemorrhage & $2 / 11(18)$ \\
$\quad$ Microhemorrhage & $7 / 11(64)$ \\
Infarction & $13 / 26(50)$ \\
$\quad$ ACA territory infarction & $0 / 13(0)$ \\
MCA territory infarction & $5 / 13(38)$ \\
PCA territory infarction & $2 / 13(15)$ \\
PICA territory infarction & $1 / 13(8)$ \\
Borderzone infarction & $1 / 13(8)$ \\
Multiterritorial infarction & $2 / 13(15)$ \\
Leukoencephalopathy & $7 / 26(27)$ \\
Multiple abnormalities (infarction + hemorrhage) & $4 / 26(15)$ \\
\hline No: & \\
\hline
\end{tabular}

Note:-SAH indicates subarachnoid hemorrahge; ACA, anterior cerebral artery; MCA, middle cerebral artery; PCA, posterior cerebral artery; PICA, posterior inferior cerebellar artery.

ancluding shortness of breath, respiratory distress, respiratory failure, and hypoxia.

Table 1: Comparison between patients with coronavirus disease 2019 with and without intracranial abnormalities

\begin{tabular}{|c|c|c|c|}
\hline & $\begin{array}{c}\text { Acute Intracranial } \\
\text { Abnormalities }\end{array}$ & $\begin{array}{c}\text { No Acute Intracranial } \\
\text { Abnormalities }\end{array}$ & $\begin{array}{c}\text { Statistical Significance } \\
\text { (P Value) }\end{array}$ \\
\hline Patients, $n$ & 26 & 124 & \\
\hline Men/women, $n$ & $16 / 10$ & $82 / 42$ & - \\
\hline Mean age, mean $\pm S D, y$ & $63.6 \pm 16$ & $64 \pm 16.5$ & $\mathrm{~ns}(P=.896)$ \\
\hline Age range, $y$ & 24-87 & $22-96$ & - \\
\hline Body mass index $\pm \mathrm{SD}, \mathrm{kg} / \mathrm{m}^{2}$ & $27.9 \pm 5.3$ & $28.5 \pm 7.7$ & $\mathrm{~ns}(P=.655)$ \\
\hline Length of stay \pm SD (days) & $24.9 \pm 12.8$ & $23.4 \pm 16.9$ & $\mathrm{~ns}(P=.091)$ \\
\hline Intensive care unit admission, $\%(n)$ & $81.5(22)$ & $62.1(77)$ & $(P=.039)^{\mathrm{a}}$ \\
\hline Intubation rate, $\%(n)$ & $77.8(21)$ & $49.2(61)$ & $(P=.004)^{\mathrm{b}}$ \\
\hline Acute kidney injury rate, \% (n) & $76.9(20)$ & $53.2(66)$ & $(P=.030)^{\mathrm{a}}$ \\
\hline Mortality rate, $\%(n)$ & $23.1(6)$ & $12.1(15)$ & $\mathrm{ns}(P=.209)$ \\
\hline
\end{tabular}

Note:-SD indicates standard deviation; ns, not significant; $y$, years.

${ }^{\mathrm{a}} \mathrm{P}<0.05$.

${ }^{\mathrm{b}} \mathrm{P}<0.01$. 

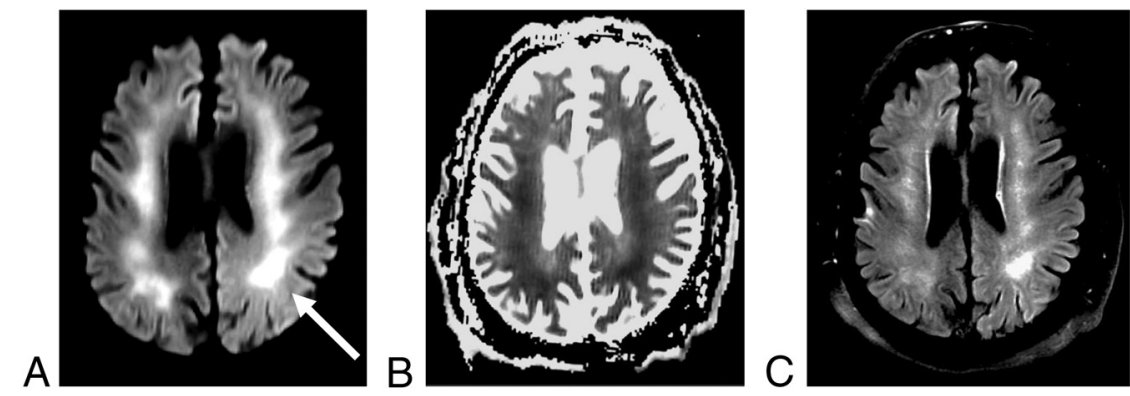

FIG 3. Leukoencephalopathy in a 61-year-old man with COVID-19. Axial DWI (A) and apparent diffusion coefficient map $(B)$ sequences are notable for extensive, confluent areas of restricted diffusion (arrow) that involve the bilateral centrum semiovale and corona radiata. (C) Corresponding T2 FLAIR hyperintensity is also seen.

For patients with intracranial abnormalities, the most common symptoms on presentation were respiratory (21/26 patients [81\%]), including shortness of breath, respiratory distress, respiratory failure, and hypoxia (Table 2 ). This was followed by fever (7/26 patients [27\%]), headache (2/26 patients [8\%]), fall $(2 / 26$ patients [8\%]), and stroke symptoms (2/26 patients [8\%]). Twelve of 26 patients (46\%) presented with multiple symptoms (Table 2). The indications for undergoing neuroimaging most commonly included altered mental status (14/26 patients [54\%]) and clinical findings consistent with stroke (8/26 patients [31\%]) (Table 2).

In the patients with positive neuroimaging studies, intracranial hemorrhage was seen in 11 patients $(42 \%)$ of the cohort (Table 2, Fig 1). Most of the patients with intracranial hemorrhage had microhemorrhages (7/11 patients [64\%]). Two patients (18\%) had SAH, and 2 had intraparenchymal hematomas. Acute or subacute infarctions were seen in 13 patients (50\%), with the most frequent territory of involvement being the MCA territory (38\%) (Table 2, Fig 2). A smaller subset of patients had infarcts in other territories, including posterior cerebral artery (2/13 patients [15\%]), posterior inferior cerebellar artery (1/13 patients $[8 \%])$, and watershed (1/13 patients [8\%]) infarcts. Two of the patients $(15 \%)$ had multiterritorial infarction. Four of the patients $(15 \%)$ had both infarction and intracranial hemorrhage. A leukoencephalopathy pattern was identified in 7 patients (27\%) of the cohort (Fig 3). None of the patients with leukoencephalopathy had intracranial hemorrhage or infarction. Similar frequencies of respiratory symptoms were noted between the groups of patients with intracranial hemorrhage and/or infarction (17/20 patients [85\%]) compared with those with leukoencephalopathy (6/7 patients [86\%]). One of the 2 patients who presented after a fall had SAH (Fig $1 B)$. The other patient who presented after a fall had acute infarction but no intracranial hemorrhage.

Statistically significant associations were found between the patients with positive neuroimaging studies and the requirement for intensive care unit admission $(P=.039)$, need for intubation $(P=.004)$, and development of acute kidney injury $(P=.030)$ (Table 1). There was no statistically significant difference in patient age, body mass index, length of stay, and mortality in patients with positive versus negative neuroimaging studies (Table 1). Comparable numbers of patients remained hospitalized as of May 19, 2020, with 52 patients $(42 \%)$ without intracranial abnormalities and 12 patients (46\%) with intracranial abnormalities still in the hospital.

\section{DISCUSSION}

The results of this study highlight a spectrum of neuroimaging findings associated with COVID-19, including intracranial hemorrhage, infarction, and leukoencephalopathy, in a 150patient cohort. In our cohort, 26 of the patients (17\%) were found to have intracranial abnormalities, of which most were related to infarction (50\%) and intracranial hemorrhage (42\%). Leukoencephalopathy was seen in $27 \%$ of the patients. Four patients (15\%) had both infarction and intracranial hemorrhage. The incidence of intracranial abnormalities in patients who underwent neuroimaging was lower than the $47 \%$ rate reported from Italy by Mahammedi et al. ${ }^{9}$ The frequency of infarctions and intracranial hemorrhages was higher in our patient cohort than the previously reported rates $(31 \%$ infarction and $6 \%$ hemorrhage), with a lower rate of leukoencephalopathy than the $35 \%$ in the study by Mahammedi et al. ${ }^{9}$ The difference in the incidence rates is likely due to various factors, among them the inclusion of spine imaging by Mahammedi et al, ${ }^{9}$ the different geographies and, therefore, populations, and possible differences in criteria used to categorize intracranial abnormalities.

Microhemorrhages have various etiologies, including cerebral amyloid angiopathy and trauma. Two of the patients had imaging studies that predated the COVID-19 pandemic, one from 2007 and the other from 2017. The patient with the more recent, 2017, previous study was 87 years old and had evidence of cerebral amyloid angiopathy on that study. However, this patient had an acute infarct on the neuroimaging obtained during admission for COVID-19. The other patient was 69 years old and had a solitary focus of microhemorrhage in 2007, with a marked increase in the number of microhemorrhages on the 2020 COVID-19-related study. The remaining 5 patients had no previous neuroimaging studies. These 5 patients spanned an age range of 41 to 60 years. Given that cerebral amyloid angiopathy is less common in this age group, we doubt that cerebral amyloid angiopathy was the cause of the hemorrhages observed in these patients with severe COVID-19 infection. The distribution of the microhemorrhages was also not specific for other causes, for example, trauma. By contrast, 1 of the patients with SAH on imaging presented after a fall, with trauma clearly acting as a confounder for the potential cause of the hemorrhage.

A significant association was demonstrated between the presence of acute abnormalities on neuroimaging studies and the severity of disease as indicated by the requirement for intensive care unit admission, the need for intubation, and development of acute kidney injury. These associations have not been previously described in the literature; however, Mao et $\mathrm{al}^{5}$ noted an association between abnormal neuroimaging studies and patients with 
severe COVID-19 lung manifestations. These data add to the growing body of literature that indicates the multisystem nature of the SARS-CoV-2 infection and its imaging manifestations. A common mechanism for multiorgan dysfunction in the disease has not yet been identified, though viral mechanisms of cellular entry and replication through exploitation of the angiotensinconverting enzyme 2 receptor are now defined. The angiotensinconverting enzyme 2 receptor is widely distributed. ${ }^{17}$ Viral invasion of endothelial cells, and an immune response to this, has been reported in diverse vascular beds in patients with COVID19. ${ }^{18}$ The SARS-CoV-2 virus has been identified in postmortem human brains, which suggests the possibility of viral tropism to cerebral vascular beds. Microvascular thrombosis and prothrombotic complications are recognized as crucial aspects of disease progression. ${ }^{19,20}$ The correlation that we describe between acute kidney injury and neuroimaging findings may indeed reflect the involvement of vascular beds in different organs, with viral or immune-mediated disruption of the neurovascular unit ${ }^{21}$ underpinning the diverse range of neuroimaging findings in these patients. These mechanisms are speculative and in need of additional study.

Previous studies on patients with COVID-19 infection have demonstrated an association between obesity and higher rates of hospitalization and mortality. ${ }^{22-25} \mathrm{We}$ investigated the possible relationship between a higher body mass index and the development of acute neurologic abnormalities in our cohort but found no significant association. One of the hypotheses for increased severity of COVID-19 infection in patients who are obese is the impediment of diaphragmatic excursion due to obesity. ${ }^{25}$ The lack of a significant association between obesity and intracranial abnormalities in our cohort may be related, in part, to this physical mechanism, as opposed to a direct, tissue-based pathologic link.

There are several limitations to this study, beyond the singlecenter, retrospective nature of it. First, we elected to use broad and intuitive measures of disease severity (intensive care unit admission, the need for intubation, acute kidney injury, and mortality) over the narrow range of reported inflammatory cytokines (D-dimer, interleukin 2, interleukin 6, etc) and other biomarkers that have been observed in patients with COVID-19 who were admitted to intensive care units. ${ }^{26}$ This decision was based on the ongoing uncertainty about the implications of these markers on disease progression and outcome. The measures that we apply are, in turn, readily applied clinically, but there may be additional associations between acute neuroimaging manifestations of COVID-19 and other organ system involvement, which are not explored here. Second, our patient cohort is relatively small, and it is possible that nonstatistically significant differences in clinical parameters, such as the mortality rate and length of stay, may achieve statistical significance with the inclusion of more patients. However, much of the literature on COVID-19 infection and associated neuroimaging findings has been in the form of case series and small cohort studies, and there is a responsibility to report observed findings at these early stages of the COVID-19 experience to guide future research directions. It is also possible that the true incidence of intracranial abnormalities is higher, given that most of the patients only had CT imaging, which is less sensitive than MR imaging to assess for subtle intracranial abnormalities, including leukoencephalopathy and low-volume infarctions. These possibilities are speculative. Third, we report findings from a defined and narrow time period in patients who were hospitalized and subsequently imaged for neurologic symptoms. The true burden of acute neuroimaging findings in the total hospitalized and nonhospitalized COVID-19 patient population, therefore, remains unknown.

\section{CONCLUSIONS}

The results of this study describe the spectrum of acute neuroimaging abnormalities observed in patients with COVID-19 that occurred at a higher frequency than reported in other cohort series. In addition, a correlation was found between acute neuro-imaging abnormalities and several clinical parameters that reflect disease severity. Clinicians treating patients with COVID-19 who develop worsened systemic disease, including acute kidney injury, with any neurologic signs or symptoms should be aware of these findings and obtain neuroimaging studies based on this association and the therapeutic implications of acute intracranial disease.

Disclosures: William A. Mehan, Jr.-UNRELATED: Consultancy: Kura Oncology, Comments: Independent reviewer of head and neck cancer imaging studies for a clinical trial; Expert Testimony: CRICO and other medical insurance companies, Comments: Expert opinion on medicolegal cases involving neuroimaging. Thabele Leslie-Mazwi-UNRELATED: Employment: Massachusetts General Hospital.

\section{REFERENCES}

1. Wang W, Tang J, Wei F. Updated understanding of the outbreak of 2019 novel coronavirus (2019-nCoV) in Wuhan, China. J Med Virol 2020;92:441-47 CrossRef Medline

2. Simpson S, Kay FU, Abbara S, et al. Radiological Society of North America expert consensus statement on reporting chest CT findings related to COVID-19. Endorsed by the Society of Thoracic Radiology, the American College of Radiology, and RSNA. Radiol Cardiothorac Imaging 2020;2:e200152 CrossRef

3. Ng M-Y, Lee EY, Yang J, et al. Imaging profile of the COVID-19 infection: radiologic findings and literature review. Radiol Cardiothorac Imaging 2020;2:e200034 CrossRef

4. Pan L, Mu M, Yang P, et al. Clinical characteristics of COVID-19 patients with digestive symptoms in Hubei, China: a descriptive, cross-sectional, multicenter study. Am J Gastroenterol 2020;115: 766-73 CrossRef Medline

5. Mao L, Jin H, Wang M, et al. Neurologic manifestations of hospitalized patients with coronavirus disease 2019 in Wuhan, China. JAMA Neurol 2020;77:1-9 CrossRef Medline

6. Poyiadji N, Shahin G, Noujaim D, et al. COVID-19-associated acute hemorrhagic necrotizing encephalopathy: CT and MRI features. Radiology 2020;296:E119-E120 CrossRef Medline

7. Ye M, Ren Y, Lv T. Encephalitis as a clinical manifestation of COVID-19. Brain Behav Immun 2020;88:945-46 CrossRef Medline

8. Moriguchi T, Harii N, Goto J, et al. A first case of meningitis/encephalitis associated with SARS-coronavirus-2. Int $J$ Infect Dis 2020;94:55-58 CrossRef Medline

9. Mahammedi A, Saba L, Vagal A, et al. Imaging in neurological disease of hospitalized COVID-19 patients: an Italian multicenter retrospective observational study. Radiology 2020 May 21. [Epub ahead of print] CrossRef Medline

10. Franceschi AM, Ahmed O, Giliberto L, et al. Hemorrhagic posterior reversible encephalopathy syndrome as a manifestation of COVID19 infection. AJNR Am J Neuroradiol 2020;41:1173-76 CrossRef Medline 
11. Radmanesh A, Derman A, Lui YW, et al. COVID-19-associated diffuse leukoencephalopathy and microhemorrhages. Radiology 2020 May 21. [Epub ahead of print ] CrossRef Medline

12. Sachs JR, Gibbs KW, Swor DE, et al. COVID-19-associated leukoencephalopathy. Radiology 2020;296:E184-E185 CrossRef Medline

13. Jain $R$, Young $M$, Dogra $S$, et al. COVID-19 related neuroimaging findings: a signal of thromboembolic complications and a strong prognostic marker of poor patient outcome. J Neurol Sci 2020; 414:116923 CrossRef Medline

14. Su H, Yang M, Wan C, et al. Renal histopathological analysis of 26 postmortem findings of patients with COVID-19 in China. Kidney Int 2020;98:219-27 CrossRef Medline

15. Okumura A, Kidokoro $H$, Tsuji $T$, et al. Differences of clinical manifestations according to the patterns of brain lesions in acute encephalopathy with reduced diffusion in the bilateral hemispheres. AJNR Am J Neuroradiol 2009;30:825-30 CrossRef Medline

16. McKinney AM, Kieffer SA, Paylor RT, et al. Acute toxic leukoencephalopathy: potential for reversibility clinically and on MRI with diffusion-weighted and FLAIR imaging. AJR Am J Roentgenol 2009;193:192-206 CrossRef Medline

17. Hamming I, Timens W, Bulthuis MLC, et al. Tissue distribution of ACE2 protein, the functional receptor for SARS coronavirus. A first step in understanding SARS pathogenesis. J Pathol 2004; 203:631-37 CrossRef Medline

18. Varga Z, Flammer AJ, Steiger P, et al. Endothelial cell infection and endotheliitis in COVID-19. Lancet 2020;395:1417-18 CrossRef Medline
19. Wang J, Hajizadeh N, Moore EE, et al. Tissue plasminogen activator (tPA) treatment for COVID-19 associated acute respiratory distress syndrome (ARDS): a case series. J Thromb Haemost 2020;18: 1752-55 CrossRef Medline

20. Oudkerk M, Büller HR, Kuijpers D, et al. Diagnosis, prevention, and treatment of thromboembolic complications in COVID19: report of the National Institute for Public Health of the Netherlands. Radiology 2020 Apr 23. [Epub ahead of print] CrossRef Medline

21. Lo EH, Broderick JP, Moskowitz MA. tPA and proteolysis in the neurovascular unit. Stroke 2004;35:354-56 CrossRef Medline

22. Zhang F, Xiong Y, Wei Y, et al. Obesity predisposes to the risk of higher mortality in young COVID-19 patients. J Med Virol 2020 May 21. [Epub ahead of print] CrossRef Medline

23. Palaiodimos L, Kokkinidis DG, Li W, et al. Severe obesity is associated with higher in-hospital mortality in a cohort of patients with COVID-19 in the Bronx, New York. Metabolism 2020;108:154262 CrossRef Medline

24. Caussy C, Pattou F, Wallet F, et al. Prevalence of obesity among adult inpatients with COVID-19 in France. Lancet Diabetes Endocrinol 2020;8:562-64 CrossRef Medline

25. Kass DA, Duggal P, Cingolani O. Obesity could shift severe COVID-19 disease to younger ages. Lancet 2020;395:1544-45 CrossRef Medline

26. Chen $\mathrm{G}, \mathrm{Wu} \mathrm{D}$, Guo W, et al. Clinical and immunologic features in severe and moderate coronavirus disease. J Clin Invest 2020; 130:2620-29 CrossRef Medline 